\title{
On the determination of the sound wave parameters during cavitation destruction of loam soil
}

\author{
Sergey Starovoytov ${ }^{1, *}$ and Valery Korotchenya ${ }^{1}$ \\ ${ }^{1}$ Federal Scientific Agroengineering Center VIM, 1st Institutsky proezd, 5, Moscow, 109428, Russia
}

\begin{abstract}
Finding the values of the frequency and sound pressure of an acoustic wave, which ensures the process of loosening loam soil, is a very important task. We assumed that the value of the average sound pressure at which the intensity of the cavitation process would be maximum is equal to twice the value of the hydrostatic pressure. The minimum radius of the formed bubble is directly proportional to the doubled surface tension of the soil solution and inversely proportional to the ultimate compressive strength of soil. The value of the ultimate compressive strength of loam soil depends on its absolute moisture content. The angular frequency of the sound field is directly proportional to the tillage depth and the ultimate compressive strength of the soil, and inversely proportional to the sound wave propagation speed and the surface tension of the soil solution. With a loam soil density $\rho=1554 \mathrm{~kg} / \mathrm{m}^{3}$ and a tillage depth $h=0.3 \mathrm{~m}$, the average sound pressure was $P_{A}=9324 \mathrm{~Pa}$. In the interval of physical ripeness of loam soil, the oscillation frequency of the sound field was in the range of 19762 to $37773 \mathrm{~s}^{-1}$.
\end{abstract}

\section{Introduction}

Tillage is one of the most important technological operations in agricultural production. Soils of the Central Federal District of Russia, the particle-size distribution of which is represented to a greater extent by loams (on average, up to 50-60\%), belong to the soils of the southern taiga zone. Such types of soils are of medium tillage complexity, and the study of feasible types of impact for breaking up the treated layer is of great significance.

Promising types of impact on the soil include its breaking up by pulses of compressed air. The frequency of impact can be commensurate with that of natural vibrations of the treated layer or an order of magnitude higher, causing a process of cavitation destruction.

The cavitation process can be of two types: hydrodynamic and acoustic $[1,2]$. Hydrodynamic cavitation occurs due to a local decrease in pressure in a fluid flow when it goes around a solid body. Hydrodynamic cavitation occurs due to a local decrease in pressure in the fluid flow during flow around a solid body. In an acoustic cavitation process that occurs when sound waves oscillate through a medium, air cavities form and collapse [3-5].

\footnotetext{
*Corresponding author: starovoitov.si@mail.ru
} 
The process of formation and collapse of cavities depends on many factors [6], including soil properties and sound wave parameters. With regard to tillage, the properties of the soil can be expressed through such physical quantities as the density of the medium, the elastic modulus of the first kind, Poisson's ratio, and wave propagation velocity. The parameters of a sound wave include frequency and sound pressure [7]. These two parameters are decisive factors in the development and design of equipment for loosening the soil by cavitation destruction. The working body for loosening the soil by cavitation destruction includes a gasjet resonator consisting of a nozzle and a resonator proper, the design features of which are dependent on the sound wave parameters.

The purpose of this study was to determine the frequency and pressure of the sound wave, which insure the breaking up of loam soil.

\section{Materials and Methods}

The intensity of the cavitation process, according to B.A. Agranat's suggestion, can be estimated with the following expression:

$$
\chi=\frac{8.14 \times\left(P_{A}-P_{O}\right)^{5 / 2} \times\left(0.2 \times P_{A}+P_{O}\right)^{7 / 2}}{P_{A}^{3} \times P_{p}^{3}}
$$

where $\chi$ is a dimensionless criterion, $P_{A}$ is the average sound pressure acting on the cavity in the compression phase $(\mathrm{Pa}), P_{O}$ is the hydrostatic pressure $(\mathrm{Pa})$, and $P_{p}$ is the saturated vapor pressure of the soil solution $(\mathrm{Pa})$.

The analysis of expression (1) showed that the criterion $\chi$ takes a maximum value under the following condition:

$$
P_{O} / P_{A}=0.5
$$

Taking into account formula (2) and the hydrostatic pressure of the soil layer, we can express the average sound pressure at which the intensity of the cavitation process will probably be maximum as follows:

$$
P_{A}=2 \times \rho \times g \times h
$$

where $\rho$ is the soil density $\left(\mathrm{kg} / \mathrm{m}^{3}\right), g$ is the gravitational acceleration $\left(\mathrm{m} / \mathrm{s}^{2}\right)$, and $h$ is the tillage depth $(\mathrm{m})$.

At the same time, the average sound pressure is equal to

$$
P_{A}=\rho \times c \times \omega \times A
$$

where $c$ is the sound wave propagation speed $(\mathrm{m} / \mathrm{s}), \omega$ is the angular frequency $\left(\mathrm{s}^{-1}\right)$, and $A$ is the sound wave displacement amplitude $(\mathrm{m})$.

The most energy-intensive deformation of the breaking up of soil is compression deformation. The compression forces are counteracted by all types of soil cohesion. These include the cohesive forces of solid particles, chemical bonds, meniscus forces of the surface tension of water, and the friction forces of interlocking and adhesion.

In order for a cavity to form in the soil solution of the treated layer during cavitation, the following condition must be met:

$$
l_{1} \leq A \leq R_{\min }
$$

where $l_{1}$ is the doubled distance between molecules (m), and $R_{\min }$ is the minimum radius of the formed bubble $(\mathrm{m})$.

It can be assumed that the sound wave displacement amplitude should be equal to the minimum radius of the formed bubble: 


$$
A=R_{\min }
$$

The minimum radius of the formed soil solution bubble is stated as follows:

$$
R_{\min }=2 \times \sigma / \sigma_{\max }
$$

where $\sigma$ is the surface tension of the soil solution $(\mathrm{N} / \mathrm{m})$, and $\sigma_{\max }$ is the maximum tensile stress $(\mathrm{Pa})$.

The surface tension of the soil solution depends on the temperature. For example, for water with an increase in temperature from $0{ }^{\circ} \mathrm{C}$ to $30{ }^{\circ} \mathrm{C}$, the surface tension is $75.64 \times 10^{-3}$ $\ldots 71.18 \times 10^{-3} \mathrm{~N} / \mathrm{m}$. At a temperature of $20^{\circ} \mathrm{C}$, the surface tension will be $72.25 \times 10^{-3} \mathrm{~N} / \mathrm{m}$.

It can also be assumed that

$$
\sigma_{\max }=\sigma_{c s}
$$

where $\sigma_{c s}$ is the ultimate compressive strength of soil $(\mathrm{Pa})$.

To determine the ultimate compressive strength of the soil, a tensile testing machine RM0.5 was used (Figure 1). The sample was loaded to the characteristic manifestations of fracture lines, at which the soil began to break up.

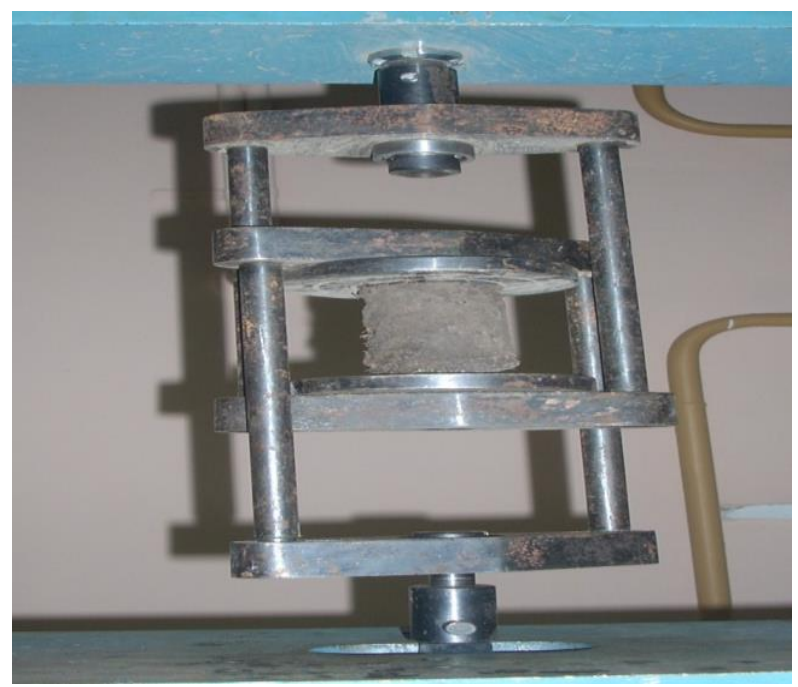

Fig. 1. Impact of the tensile testing machine RM-0.5 on a soil sample.

As a result of testing, the following regression equation was obtained

$$
\sigma_{c s}=\left(-0.0003 \times \omega_{a}^{2}+0.0015 \times \omega_{a}+0.252\right) \times 10^{6}
$$

where $\omega_{a}$ is the absolute moisture content (\%).

Thus, the angular frequency of the sound field is as follows:

$$
\omega=\frac{2 \times g \times h \times \sigma_{c S}}{c \times \sigma}
$$

In media, longitudinal and transverse waves can propagate. A longitudinal wave is a wave during the propagation of which the particles of the medium are displaced in the direction of the wave propagation. The cause of the longitudinal wave is compression/tension deformations. In liquids and gases, such deformation is accompanied by rarefaction or compression of the medium particles. Longitudinal waves can propagate in any mediumsolid, liquid, and gaseous. 
In turn, a transverse wave is a wave during the propagation of which the particles of the medium are displaced in a direction perpendicular to the propagation of the wave. The cause of the transverse wave is the shear deformation of one layer of the medium relative to another. When a transverse wave propagates in a medium, crests and troughs are formed. Liquids and gases, unlike solids, do not have resilience with respect to the shear of the layers, i.e. do not resist shape change. Therefore, transverse waves can propagate only in solids.

Let us assume that the treated soil layer is an infinite solid body, in which only longitudinal waves propagate. Then, the propagation velocity of the longitudinal wave is equal to

$$
c=\sqrt{\frac{E \times(1-\mu)}{\rho \times(1+\mu) \times(1-2 \times \mu)}}
$$

where $E$ is the elastic modulus of soil of the first kind ( $\mathrm{Pa})$, and $\mu$ is Poisson's ratio.

The above two parameters reflect the soil of a certain particle-size distribution and absolute moisture content.

To determine the elastic modulus of the first kind, the Kushnarev installation was used (Figure 2). The device includes a metal support 1, pins 2, and an upper plate 5 . The two pins 2 , which serve as guides for the upper plate 5, are screwed into the metal support 1 . A coil 6 is placed on the upper plate 5 . The prepared soil sample 4 is fixed in a ring 3 , which, in turn, fixed motionlessly on the metal support 1 . On the upper edge of the soil sample, there is a support ring (not shown) on which a permanent magnet 7 is located. The permanent magnet 7 enters the coil 6 of the upper plate 5 .

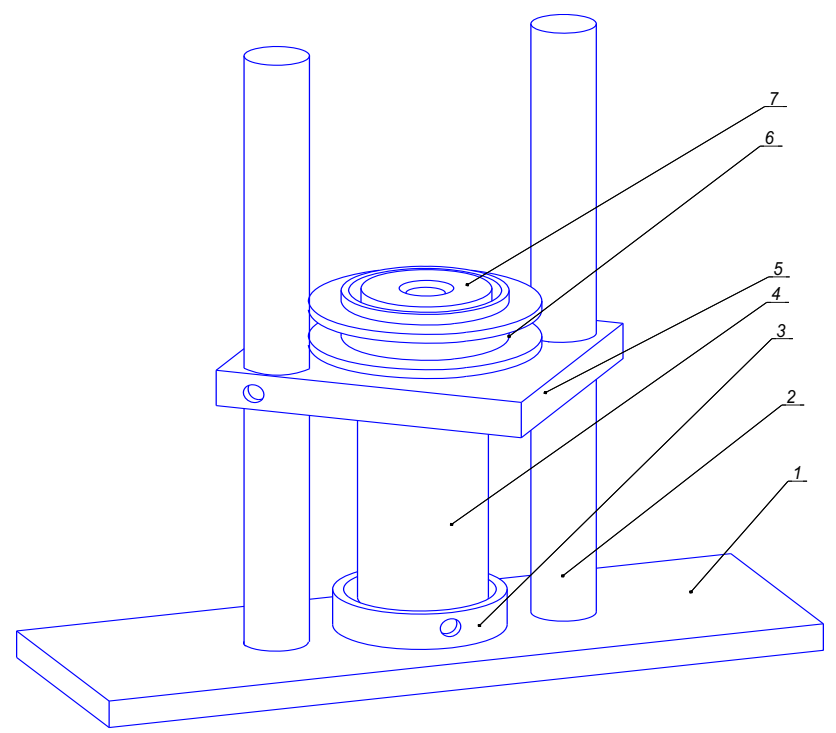

Fig. 2. Device for determining the technical vibration frequency.

Under impulse loading, the soil sample is initially compressed and, subsequently, oscillates, which leads to the creation of an EMF in the coil 6 . This signal is recorded by the $\mathrm{ADC}$ and displayed on the computer screen, where the oscillation curve of the upper end and the graph of the correspondence between the amplitude and frequency are shown. Having determined the technical vibration frequency, we determine the elastic modulus of the first kind.

The elastic modulus of the first kind [8] is equal to

$$
E=-47998 \times \omega_{a}^{2}+1520917 \times \omega_{a}-7.7 \times 10^{6}
$$


To determine Poisson's ratio, a stabilometer was used (Figure 3).

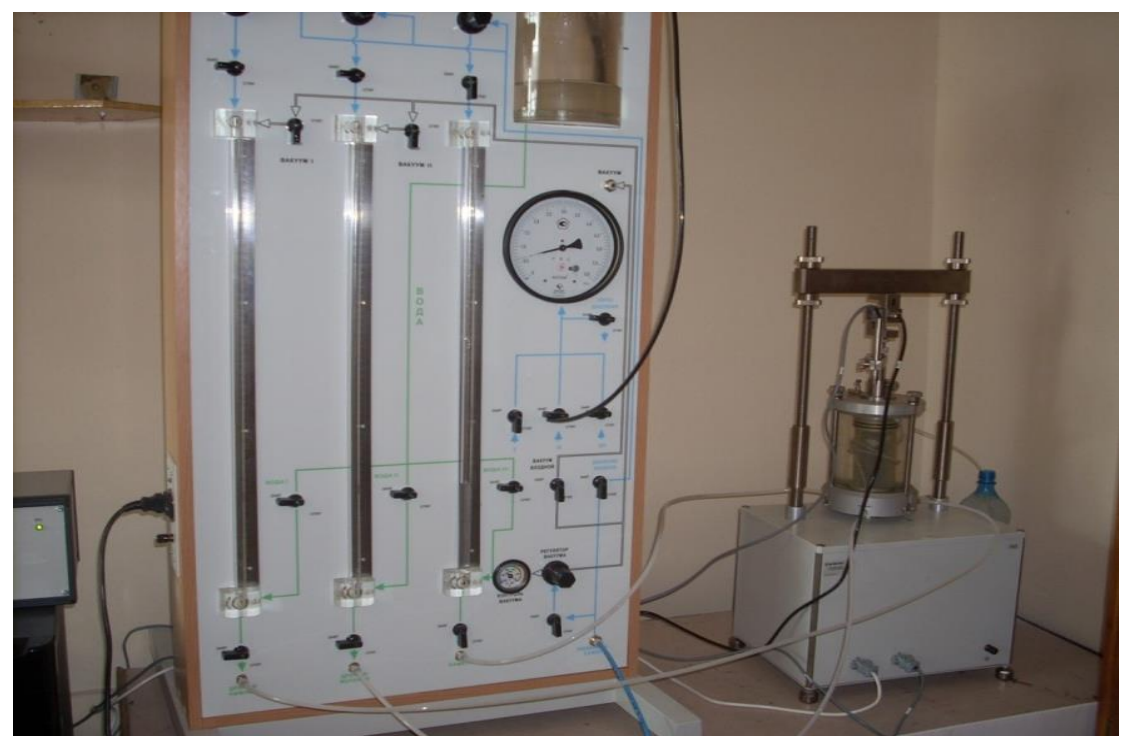

Fig. 3. Stabilometer compression chamber.

Poisson's ratio can be expressed in terms of the absolute moisture content as follows:

$$
\mu=-0.002 \times \omega_{a}^{2}+0.062 \times \omega_{a}-0.008
$$

\section{Results}

It has been established that the value of the average sound pressure is in direct proportion to the density of the soil and the depth of cultivation (Figure 4). When the depth of cultivation changes in the interval of $0.05 \mathrm{~m} \ldots 0.31 \mathrm{~m}$, the sound pressure is in the range of $1554 \mathrm{~Pa} \ldots$ $9634 \mathrm{~Pa}$. With a loam soil density $\rho=1554 \mathrm{~kg} / \mathrm{m}^{3}$ and a tillage depth $h=0.3 \mathrm{~m}$, the average sound pressure was $P_{A}=9324 \mathrm{~Pa}$.

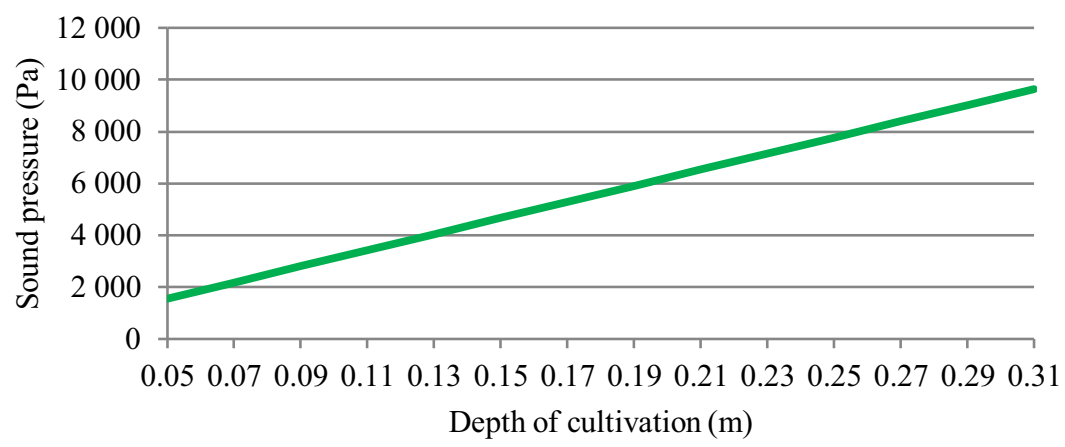

Fig. 4. Relationship between the sound pressure and the depth of cultivation.

Figure 5 shows the relationship between the oscillation frequency of the sound field and the absolute moisture content of loam soil. In the interval of physical ripeness of loam soil, the oscillation frequency of the sound field was in the range of 19762 to $37773 \mathrm{~s}^{-1}$. 


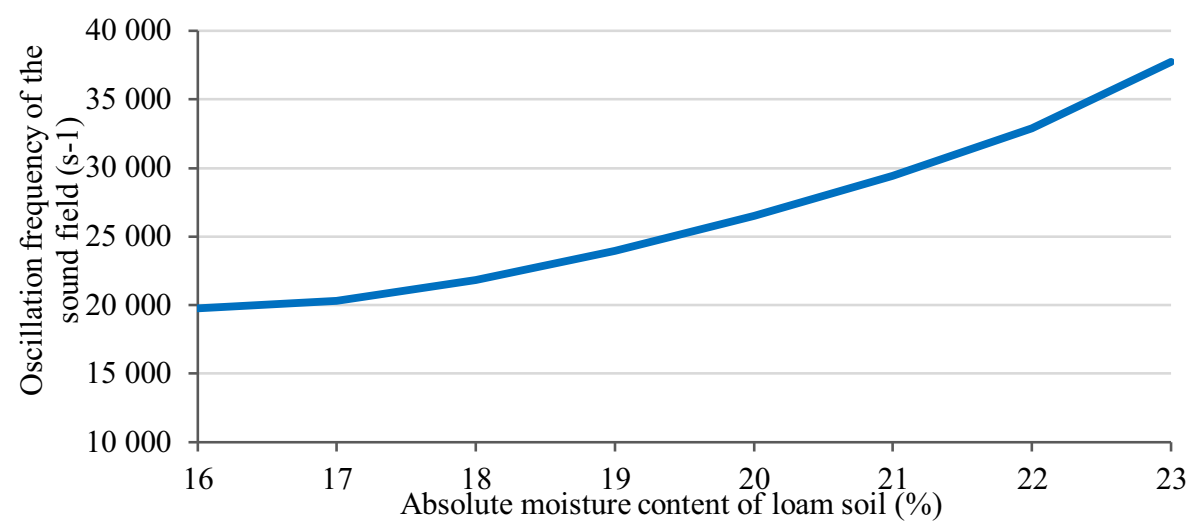

Fig. 5. Relationship between the oscillation frequency of the sound field and the absolute moisture content of loam soil.

The studied quantity assumes a minimum value of $19762 \mathrm{~s}^{-1}$ at an absolute moisture content of $16 \%$, and a maximum value of $37773 \mathrm{~s}^{-1}$ at that of $23 \%$. The following has been established: with an increase in the absolute moisture content, the frequency of the sound field increases in a parabolic relationship.

\section{Discussion}

Ultrasonic impact is widely used in manufacturing: for high-quality spraying of liquids, manual or automated cutting of materials, ignition of fuels, processing of solid materials, cleaning of solid deposits from pipe walls and openings of technical systems in the oil industry [9], assessment of the aggregate state of soil particles [10], etc.

The defining characteristic of the operability of the gas-jet resonator is the vibration frequency of the sound wave, which, in turn, depends on the absolute moisture content of the cultivated loam soil.

The investigated magnitude takes the minimum value of $19762 \mathrm{~s}-1$ at an absolute moisture content of $16 \%$, and the maximum value of $37773 \mathrm{~s}-1$ is reached at an absolute moisture content of $23 \%$. It was found that with an increase in the value of absolute moisture content, the frequency of the sound field increases in a parabolic relationship.

This interval of sound frequency is comparable with the studies of many scientists [1115], in particular the one of Matteo Erriu et al. [11], where the effect of ultrasound on microorganisms was examined.

With an absolute moisture content of loam soil of $18 \%$, the frequency of vibration of a sound wave is $21000 \mathrm{~Hz}$. This parameter is comparable with the results of Chinese scientists [16].

The value of the average sound pressure is less than the value examined for deagglomeration of liquid aluminium [17].

\section{Conclusions}

We developed a mathematical model that established the relationship between the parameters of the sound wave, which insures the breaking up of the treated layer, and the absolute moisture content of soil. 
According to the results obtained when simulating the process of cavitation destruction of the treated medium, with a density of loam soil $\rho=1554 \mathrm{~kg} / \mathrm{m}^{3}$ and a tillage depth $h=$ $0.3 \mathrm{~m}$, the average sound pressure was $P_{A}=9324 \mathrm{~Pa}$.

We established that in the interval of physical ripeness of loam soil, which corresponds to $16-23 \%$ of absolute moisture content, the oscillation frequency of the sound field was between 19762 and $37773 \mathrm{~s}^{-1}$.

The obtained relationship between the oscillation frequency of the sound field and the absolute moisture content of loam soil will make it possible to calculate the geometric parameters of a gas-jet radiator, which includes a nozzle and a resonator.

\section{References}

1. H. Muramatsu Mizutani, T. Saito, Chem. Eng. Sci. 206, 476-488 (2019) doi:10.1016/j.ces.2019.04.053

2. H. Tsuge, Micro- and Nanobubbles: Fundamentals and Applications (2014) doi:10.1201/b17278

3. Ch.E. Brennen, Cavitation and Bubble Dynamics doi:10.1017/cbo9781107338760

4. R.P. Taleyarkhan, C.D. West, R.T. Lahey, Jr., R.I. Nigmatulin, R.C. Block, Y. Xu, Phys. Rev. Lett. 96, 034301 (2006) doi:10.1103/PhysRevLett.96.034301

5. Kyuichi Yasui, Acoustic Cavitation and Bubble Dynamics (2018) doi:10.1007/978-3319-68237-2

6. Zhengwei Li, Zhiwu Xu, Degang Zhao, Shu Chen, Jiuchun Yan, Ultrason. Sonochem. 71, 105356 (2021) doi:10.1016/j.ultsonch.2020.105356

7. R.I. Li, A.A. Kolesnikov, Increasing the efficiency of resources use in the production of agricultural products - new technologies and machinery of a new generation for crop and livestock production: Collection of scientific papers of the XVII International scientific and practical conference (2013)

8. V.E. Torikov, S.I. Starovojtov, N.N. Chemisov, Zemledelie 8, 19-21 (2016)

9. Habiba Lais, Premesh S. Lowe, Tat-Hean Gan, Luiz C. Wrobel, Ultrason. Sonochem. 45, 7-16 (2018) doi:10.1016/j.ultsonch.2018.02.045

10. J. Schomakers, A. Mentler, T. Steurer, A. Klik, H. Mayer, Int. Agrophys. 25, 165-172 (2011)

11. M. Erriu, G. Genta, D. Madonna Ripa et al., Measurement 80, 148-153 (2016) doi:10.1016/j.measurement.2015.11.009

12. J. Schomakers, F. Zehetner, A. Mentler, F. Ottner, H. Mayer, Int. Agrophys. 29, 501508 (2015) doi:10.1515/intag-2015-0057

13. C. Vanhille, C. Campos-Pozuelo, C. Granger, B. Dubus, Phys. Procedia 70, 1070-1073 (2015) doi:10.1016/j.phpro.2015.08.228

14. Younggyu Son, Yunsung No, Jeonggwan Kim, Ultrason. Sonochem. 65, 105065 (2020) doi:10.1016/j.ultsonch.2020.105065

15. V. Salinas, Y. Vargas, O. Louisnard, L. Gaete, Ultrason. Sonochem. 22, 227-234 (2015) doi:10.1016/j.ultsonch.2014.07.007

16. Wang Jiasheng, Kuang Jun, Wang Dongwei et al., China Patent CN108029252A, The bidirectional crossed vibration soil excavating gear of ultrasonic wave (2017)

17. G.S. Bruno Lebon, I. Tzanakis, K. Pericleous, D. Eskin, Ultrason. Sonochem. 42, 411421 (2018) doi:10.1016/j.ultsonch.2017.12.002 